\title{
Disseminated Intravascular Coagulation, Hemoperitoneum, and Reversible Ischemic Neurological Deficit Complicating Anaphylaxis to Prophylactic Antibiotics during Cesarean Delivery: A Case Report and Review of Literature
}

\author{
Mostafa A. Borahay, M.D., ${ }^{1}$ Hassan M. Harirah, M.D., ${ }^{1}$ Gayle Olson, M.D., 1 \\ Gokhan S. Kilic, M.D., ${ }^{1}$ Sinem Karipcin, M.D., ${ }^{1}$ and Gary D.V. Hankins, M.D. ${ }^{1}$
}

Routine use of prophylactic antibiotics reduces the risk of postcesarean fever and infections by over $50 \%$ in both nonelective and elective (scheduled) procedures. Although anaphylaxis to prophylactic antibiotics is rare, potentially fatal complications might occur. Herein, we present a case where disseminated intravascular coagulation and reversible ischemic neurological deficit complicated anaphylactic reactions to prophylactic antibiotics administered during cesarean delivery. A 27-year-old gravida 9, para 7 at $39^{2} / 7$ weeks underwent elective repeat cesarean delivery and bilateral tubal ligation. Her surgery was complicated by intraoperative hypotension, generalized itching, and urticarial skin rash consistent with anaphylactic reaction upon administering prophylactic cefazolin. In the recovery room, she continued to be hemodynamically unstable despite energetic resuscitation. Hemoperitoneum was suspected, and laboratory evaluation indicated disseminated intravascular coagulation. Abdominal exploration revealed massive hemoperitoneum, but there was no source of active bleeding noted. The postoperative course was complicated by reversible ischemic neurological deficit, which resolved on expectant management. Disseminated intravascular coagulation and reversible ischemic neurological deficit may complicate anaphylactic reaction to prophylactic antibiotics administered during cesarean delivery. Immediate recognition and intervention is crucial for a successful outcome.

KEYWORDS: Disseminated intravascular coagulation, anaphylaxis, anaphylactic reactions, reversible ischemic neurological deficit, cesarean delivery

\footnotetext{
${ }^{1}$ Department of Obstetrics and Gynecology, The University of Texas Medical Branch, Galveston, Texas.

Address for correspondence and reprint requests: Mostafa Borahay, M.D., Departments of Obstetrics and Gynecology, The University of Texas Medical Branch, Galveston, TX 77555-0587 (e-mail: maboraha $@$ utmb.edu).

Am J Perinatol Rep 2011;1:15-20. Copyright (C) 2011 by Thieme
}

Medical Publishers, Inc., 333 Seventh Avenue, New York, NY 10001, USA. Tel: +1(212) 584-4662.

Received: November 3, 2010. Accepted: November 3, 2010. Published online: January 24, 2011.

DOI: http://dx.doi.org/10.1055/s-0030-1271219.

ISSN 2157-6998. 
Infection remains among the top five causes of pregnancy-related mortality and accounts for a disproportionate contribution to maternal morbidity, both in the United States and around the world. ${ }^{1}$ Cesarean delivery is the single most important risk factor for postpartum infection. Women who deliver via cesarean have 5- to 20-fold increased risk for postpartum infection compared with those delivered vaginally. ${ }^{2}$ Routine use of prophylactic antibiotics reduces the risk of postcesarean fever and infections by over $50 \%$ in both nonelective and elective (scheduled) procedures. ${ }^{2,3}$ Given these clear benefits, administration of prophylactic antibiotics became the standard of care in modern obstetric practice. Our case describes a patient who developed intraoperative anaphylaxis to prophylactic antibiotics during cesarean delivery. Her postoperative course was complicated with hemoperitoneum, disseminated intravascular coagulation (DIC), and reversible ischemic neurological deficit. Review of the literature revealed few cases of anaphylaxis during cesarean section. To our knowledge, this is the first case of anaphylaxis during cesarean section followed by this set of complications. Herein, we will describe the case and review relevant literature.

\section{CASE REPORT}

A 27 -year-old woman (gravida 9, para 7) at $39^{2} / 7$ weeks' gestation presented for scheduled repeat cesarean delivery and bilateral tubal ligation. Her past medical history was significant for prior allergic reaction to iodine and intravenous (IV) contrast media (anaphylaxis). Her antenatal care was significant for iron-deficiency anemia. On presentation, her vital signs were blood pressure (BP) 133/83 mm Hg; heart rate (HR) 82/min; temperature $36.6^{\circ} \mathrm{C}$, and respiratory rate $20 / \mathrm{min}$. Physical examination revealed mild pallor and term gestation. Laboratory evaluation showed hemoglobin $8.3 \mathrm{~g} / \mathrm{dL}$, hematocrit $25.9 \%$, and platelet count $232,000 / \mathrm{mL}$. Given her moderate anemia, 2 U packed red blood cells were crossmatched prior to surgery.

In the operating room, spinal anesthesia was administered without difficulty, and cefazolin $1 \mathrm{~g}$ was given intravenously after umbilical cord clamping. Following administration of antibiotic, the patient's BP dropped to $90 / 50 \mathrm{~mm} \mathrm{Hg}$, but her HR remained stable at $70 / \mathrm{min}$ and $\mathrm{O}_{2}$ saturation at $100 \%$. The initial impression was spinal hypotension, and IV fluids and vasopressors (ephedrine sulfate $40 \mathrm{mg}$ intramuscularly, phenylephrine $1 \mathrm{mg}$, followed by $2 \mathrm{mg} \mathrm{IV}$ ) were administered. Five minutes later, BP dropped further to $55 / 25 \mathrm{~mm} \mathrm{Hg}$, but $\mathrm{HR}$ and $\mathrm{O}_{2}$ saturation remained stable at $75 / \mathrm{min}$ and $100 \%$, respectively. The patient remained fully conscious and complained of generalized itching. Anaphylaxis was suspected, and an epinephrine drip was initiated at $0.03 \mu \mathrm{g} / \mathrm{kg} / \mathrm{min}$ and then titrated to effective dose of $0.1 \mu \mathrm{g} / \mathrm{kg} / \mathrm{min}$ until $\mathrm{BP}$ was restored to
120/70 mm Hg. Hydrocortisone $100 \mathrm{mg}$, diphenhydramine $50 \mathrm{mg}$, and famotidine $20 \mathrm{mg}$ were also administered intravenously. Surgery, including closure of hysterotomy and bilateral tubal ligation, was completed, and hemostasis was secured. Generalized peau d'orange rash was noted upon closure if the skin. After surgery, the patient was taken to the postanesthetic care unit (PACU) for close observation.

In the PACU, the patient was hemodynamically stable on continuous epinephrine $0.1 \mu \mathrm{g} / \mathrm{kg} / \mathrm{min}$ infusion. However, after 150 minutes, her BP dropped again to $70 / 45 \mathrm{~mm} \mathrm{Hg}$, and HR increased to $140 / \mathrm{min}$. No vaginal bleeding was noted, but her abdomen was notably distended. Bedside ultrasonography showed free intraabdominal fluid. Laboratory evaluation revealed DIC; drop of hematocrit 20.4\%, and platelet count 103,000/ $\mathrm{mL}$; elevated prothrombin time 30.9 seconds; international normalized ratio of 3 , and activated partial thromboplastin time (aPTT) 57 seconds; low fibrinogen $62 \mathrm{mg} /$ $\mathrm{dL}$; and elevated fibrin degradation products to $>20 \mu \mathrm{g} /$ $\mathrm{mL}$. Packed red blood cells and fresh frozen plasma were transfused in addition to crystalloid and colloid fluids. Due the continued hemodynamic instability, the patient was taken back to the operating room for an exploratory laparotomy. Massive hemoperitoneum with $\sim 4 \mathrm{~L}$ of blood was noted. Hysterotomy was inspected. No active bleeders were evident, but generalized oozing was noted. Further exploration revealed intact liver, kidneys, bowel, and omentum. Both uterine arteries and infundibulopelvic ligaments were ligated, and cryoprecipitate and thrombin were applied locally to control areas of oozing. After securing hemostasis, fascia was closed, subcutaneous space was packed, and skin incision was left open for delayed primary closure.

The postoperative course after laparotomy was uneventful, and the patient was maintained on steroids, H1- and H2-blockers, and close observation. The clinical picture of DIC resolved within the first 24 hours. She remained intubated in the surgical intensive care unit for a total of 48 hours before transfer to the general floor. On the fifth postoperative day, the patient complained of blurry vision in her left eye as well as decreased sensation and weakness of her right arm. Brain magnetic resonance imaging with contrast revealed no abnormalities, and within 4 days, these neurological manifestations completely resolved. Given the completely reversible nature of these abnormalities and negative brain imaging, the impression was reversible ischemic neurological deficit. The patient continued to show satisfactory improvement and was discharged home on the tenth postoperative day in good condition.

\section{DISCUSSION}

Anaphylaxis, as recently defined by the National Institute of Allergy and Infectious Disease and Food Allergy 
and Anaphylaxis Network symposium, is "a serious allergic reaction that is rapid in onset and may cause death." ${ }^{4}$ The lifetime prevalence of anaphylaxis from all triggers is estimated to be 0.05 to $2 \%$. The most frequently identified triggers for anaphylaxis include foods (especially peanuts and tree nuts), drugs (antibiotics, vaccines, medications, and anesthetics), insect venoms, latex, and allergen immunotherapy injections. There are also a significant number of anaphylaxis cases reported for which there is no cause identified (idiopathic anaphylaxis). ${ }^{5}$

Although anaphylaxis is a rare event during pregnancy, it has the potential to lead to serious maternal and/ or fetal morbidity. In labor and delivery units, anaphylaxis is mainly caused by $\beta$-lactam antibiotics given for group B streptococcal prophylaxis. ${ }^{6}$ Mulla et $\mathrm{al}^{7}$ studied the prevalence of anaphylaxis in obstetric patients using a statewide hospital discharge database of the state of Texas during 2 years (2004 and 2005). Of 705,183 deliveries, they found 19 cases, with a prevalence of 2.7 cases per 100,000 deliveries. $\beta$-Lactam antibiotics (specifically, a penicillin or a cephalosporin) were the anaphylactic trigger in 11 patients. Because antibiotics are frequently used in obstetrics patients, obstetricians are prone to encounter such anaphylactic reactions. Therefore, they should be familiar with criteria for diagnosis of anaphylaxis and its management. These criteria (Table 1) were proposed to facilitate the immediate recognition of anaphylaxis and to standardize its diagnosis as well as providing a description for epidemiologic and research purposes. These criteria are likely to capture anaphylactic reactions in more than $95 \%$ of cases. Of note, the first criterion alone (that includes skin and/or mucous membrane involvement) captures $80 \%$ of cases, whereas $~ 20 \%$ of cases lack such involvement. ${ }^{4}$
Sudden intrapartum cardiopulmonary distress is an acute emergency, and rapid assessment and intervention is crucial. In our case, the main differential diagnoses include anaphylaxis and amniotic fluid embolism. Because both conditions present with very similar manifestations, in many cases it is difficult to differentiate intrapartum anaphylaxis from amniotic fluid embolism. Data from the national amniotic fluid embolus registry suggest that amniotic fluid embolism is more similar to anaphylaxis than to an embolism per se, ${ }^{8}$ and the term anaphylactoid syndrome of pregnancy has been suggested. The striking similarities between clinical and hemodynamic findings in amniotic fluid embolism and anaphylaxis suggest a common pathophysiological mechanism for all these conditions. Moreover, similarities of both conditions include laboratory findings. Serum tryptase level, usually used as a marker for anaphylaxis, was noted similarly elevated in a case of amniotic fluid embolism. ${ }^{9}$ The immediate differentiation of both conditions comes second after prompt management including hemodynamic support and aggressive resuscitation.

In our case, the diagnosis of anaphylaxis was supported by the presence of cutaneous involvement with generalized itching and urticarial rash and the absence of hypoxemia. The potential trigger factors of anaphylaxis include administration of antibiotics, anesthetic agents, or contact with latex. However, there was a direct temporal relation of anaphylaxis upon the administration of cefazolin antibiotic after delivering the infant and clamping the umbilical cord. This chronologic relation makes latex allergy unlikely as anaphylaxis to latex usually occurs at the beginning of surgery upon patient's contact with latex products such as surgical gloves.

\section{Table 1 Clinical Criteria for Diagnosing Anaphylaxis}

\section{Anaphylaxis is highly likely when any one of the following three criteria is fulfilled:}

1. Acute onset of an illness (minutes to several hours) with involvement of the skin, mucosal tissue, or both

(e.g., generalized hives, pruritus or flushing, swollen lips, tongue, or uvula)

and at least one of the following

a. Respiratory compromise (e.g., dyspnea, wheeze-bronchospasm, stridor, reduced PEF, hypoxemia)

b. Reduced BP or associated symptoms of end-organ dysfunction (e.g., hypotonia [collapse], syncope, incontinence)

2. Two or more of the following that occur rapidly after exposure to a likely allergen for that patient (minutes to several hours)

a. Involvement of the skin-mucosal tissue (e.g., generalized hives, itch-flush, swollen lips, tongue, uvula)

b. Respiratory compromise (e.g., dyspnea, wheeze-bronchospasm, stridor, reduced PEF, hypoxemia)

c. Reduced BP or associated symptoms (e.g., hypotonia [collapse], syncope, incontinence)

d. Persistent gastrointestinal symptoms (e.g., crampy abdominal pain, vomiting)

3. Reduced BP after exposure to known allergen for that patient (minutes to several hours)

a. Infants and children: low systolic BP (age-specific*) or greater than $30 \%$ decrease in systolic BP

b. Adults: systolic BP of less than $90 \mathrm{~mm} \mathrm{Hg}$ or greater than $30 \%$ decrease from that person's baseline

*Low systolic BP for children is defined as less than $70 \mathrm{~mm} \mathrm{Hg}$ from 1 month to 1 year, less than $(70 \mathrm{~mm} \mathrm{Hg}+$ [2 $\times 3$ age]) from 1 to 10 years, and less than $90 \mathrm{~mm} \mathrm{Hg}$ from 11 to 17 years.

Reprinted from Sampson HA, et al. Second symposium on the definition and management of anaphylaxis: Summary report-Second National Institute of Allergy and Infectious Disease/Food Allergy and Anaphylaxis Network symposium. J Allergy Clin Immunol 2006;117(2):391-397, with permission from Elsevier.

$\mathrm{BP}$, blood pressure; PEF, peak expiratory flow. 
In this case, anaphylaxis was followed by two rare complications: DIC and reversible ischemic neurological deficit. Although the relation between anaphylaxis and DIC has been described in animal trials, there is a scarcity of published cases in humans. Review of the literature revealed only two such cases. Inal et $\mathrm{al}^{10}$ described a 16-year-old girl who underwent burn scar deformity reconstruction with insertion of latex tissue expanders. This was followed by late-onset hypotension, thrombocytopenia, DIC, and pulmonary edema. Lombardini et $\mathrm{al}^{11}$ reported a 19 -year-old woman who sustained anaphylactic shock due to a wasp sting. She subsequently had prolonged prothrombin time, prolonged aPTT, and a low fibrinogen level, but a normal platelet count. She had no bleeding, and these laboratory abnormalities normalized in 24 hours.

Choi et $\mathrm{al}^{12}$ used a mouse model to investigate the role of platelet-activating factor (PAF) as the potential link between anaphylaxis and DIC at the molecular level. Induction of active systemic anaphylaxis resulted in the development of DIC manifestations, which were prevented by pretreatment with platelet-activating factor antagonist (BN 50739). Moreover, the same changes were produced by a bolus injection of PAF. These findings strongly suggest that DIC can occur in anaphylaxis, and PAF plays a pivotal role in the development of DIC in anaphylaxis.

As with the treatment of any critically ill patient, the treatment of anaphylaxis begins with a rapid assessment and maintenance of airway, breathing, and circulation. When a patient fulfills any of the three criteria of anaphylaxis as mentioned in Table 1 , the patient should receive epinephrine immediately as the treatment of choice. Subsequent interventions are determined on the basis of the clinical course and response to epinephrine. Aqueous epinephrine, $0.01 \mathrm{mg} / \mathrm{kg}$ (maximum dose, 0.5 $\mathrm{mg}$ ) administered intramuscularly every 5 to 15 minutes as necessary, is the recommended dosage for controlling symptoms and maintaining blood pressure. The 5 -minute interval between injections can be liberalized to permit more frequent injections if deemed necessary. Intravenous epinephrine is an option for patients with severe hypotension or cardiac arrest unresponsive to intramuscular doses of epinephrine and fluid resuscitation. Although there is no precisely established dosage or regimen for intravenous epinephrine in anaphylaxis, 5- to $10-\mu \mathrm{g}$ intravenous bolus $(0.2 \mu \mathrm{g} / \mathrm{kg})$ doses for hypotension and 0.1 to $0.5 \mathrm{mg}$ IV in the presence of cardiovascular collapse have been suggested. ${ }^{4}$ It is important to recognize the potential for lethal arrhythmias when administering intravenous epinephrine; therefore, continuous cardiac monitoring is recommended. Continuous low-dose epinephrine infusion might represent the safest and most effective form of intravenous administration since the dose can be titrated to the desired effect. Low-dose intravenous epinephrine infusion has been successfully used in anaphylaxis during labor and delivery without untoward fetal effects. ${ }^{7}$ It is of paramount importance to note that intravenous injection of epinephrine should only be done using dilute $0.1 \mathrm{mg} / \mathrm{mL}(1: 10,000)$ solution, whereas intramuscular injection is done using the rather concentrated $1 \mathrm{mg} / \mathrm{mL}(1: 1000)$ solution. Mistakes in this regard can have catastrophic consequences. ${ }^{13}$

Patients who remain hypotensive despite epinephrine should have aggressive fluid resuscitation. Large volumes of crystalloid might be needed in the first 5 to 10 minutes; in severe reactions with hypotension, up to $35 \%$ of the blood volume might extravasate in the first 10 minutes, and vasodilatation can cause pooling, with even more reduction in the effective blood volume and, thus, distributive shock. Potent vasopressors, such as noradrenaline, vasopressin, or metaraminol, might be required to overcome vasodilatation if epinephrine and fluid resuscitation have failed to maintain a systolic blood pressure of greater than $90 \mathrm{~mm} \mathrm{Hg}$. Antihistamines (H1- and H2-antagonists) are slower in onset of action than epinephrine, have little effect on blood pressure, and should be considered a second-line treatment for anaphylaxis. The effectiveness of corticosteroids in anaphylaxis has never been determined in placebo-controlled trials. However, their usefulness in other allergic diseases has led to their incorporation into anaphylaxis management. Because the onset of action is slow, steroids are not useful in the acute management stage. It has been suggested that their use might prevent a protracted or biphasic reaction. If given, the dosing of intravenous corticosteroids should be equivalent to 1.0 to $2.0 \mathrm{mg} / \mathrm{kg}$ per dose of methylprednisolone every 6 hours. ${ }^{4}$

Reversible ischemic neurological deficit has been defined as a sudden, focal neurological deficit of a presumed vascular origin lasting 24 hours to 7 days. Recently, there has been a shift toward tissue diagnosis rather than time diagnosis with high-resolution computed tomography and diffusion-weighted magnetic resonance imaging studies used to detect even very small infarctions. ${ }^{14}$ In our case, neurological deficit is thought to have been secondary to the combination of hypotension and DIC. These neurological deficits completely resolved after resuscitation and expectant management. Because these ischemic changes can progress and become irreversible, obstetricians should be aware and promptly identify and treat such serious conditions.

In summary, this case highlights the importance of prompt recognition and management of anaphylaxis by obstetricians. Aggressive treatment of anaphylactic reactions can prevent other serious complications, including DIC and neurological vascular sequelae. Finally, a multidisciplinary approach with appropriate consultation is important in the management of these cases. 


\section{REFERENCES}

1. Berg CJ, Chang J, Callaghan WM, Whitehead SJ. Pregnancyrelated mortality in the United States, 1991-1997. Obstet Gynecol 2003;101:289-296

2. Smaill FM, Gyte GM. Antibiotic prophylaxis versus no prophylaxis for preventing infection after cesarean section. Cochrane Database Syst Rev 2010;(1):CD007482

3. Chelmow D, Ruehli MS, Huang E. Prophylactic use of antibiotics for nonlaboring patients undergoing cesarean delivery with intact membranes: a meta-analysis. Am J Obstet Gynecol 2001;184:656-661

4. Sampson HA, Muñoz-Furlong A, Campbell RL, et al. Second symposium on the definition and management of anaphylaxis: summary report-Second National Institute of Allergy and Infectious Disease/Food Allergy and Anaphylaxis Network symposium. J Allergy Clin Immunol 2006;117: 391-397

5. Peavy RD, Metcalfe DD. Understanding the mechanisms of anaphylaxis. Curr Opin Allergy Clin Immunol 2008;8: 310-315

6. Gei AF, Pacheco LD, Vanhook JW, Hankins GD. The use of a continuous infusion of epinephrine for anaphylactic shock during labor. Obstet Gynecol 2003;102:1332-1335

7. Mulla ZD, Ebrahim MS, Gonzalez JL. Anaphylaxis in the obstetric patient: analysis of a statewide hospital discharge database. Ann Allergy Asthma Immunol 2010;104:55-59

8. Clark SL, Hankins GD, Dudley DA, Dildy GA, Porter TF. Amniotic fluid embolism: analysis of the national registry. Am J Obstet Gynecol 1995;172(4 Pt 1):1158-1167; discussion 1167-1169
9. Farrar SC, Gherman RB. Serum tryptase analysis in a woman with amniotic fluid embolism. A case report. J Reprod Med 2001;46:926-928

10. Inal MT, Memis D, Top H, et al. Late-onset pulmonary edema and disseminated intravascular coagulation due to latex anaphylaxis. Aesthetic Plast Surg 2010;34:394-396

11. Lombardini C, Helia RE, Boehlen F, Merlani P. "Heparinization" and hyperfibrinogenolysis by wasp sting. Am J Emerg Med 2009;27:1176-, e1-e3

12. Choi IH, Ha TY, Lee DG, et al. Occurrence of disseminated intravascular coagulation (DIC) in active systemic anaphylaxis: role of platelet-activating factor. Clin Exp Immunol 1995;100:390-394

13. Simons FE. Anaphylaxis: recent advances in assessment and treatment. J Allergy Clin Immunol 2009;124:625-636; quiz 637-638

14. Easton JD, Saver JL, Albers GW, et al; American Heart Association; American Stroke Association Stroke Council; Council on Cardiovascular Surgery and Anesthesia; Council on Cardiovascular Radiology and Intervention; Council on Cardiovascular Nursing; Interdisciplinary Council on Peripheral Vascular Disease. Definition and evaluation of transient ischemic attack: a scientific statement for healthcare professionals from the American Heart Association/American Stroke Association Stroke Council; Council on Cardiovascular Surgery and Anesthesia; Council on Cardiovascular Radiology and Intervention; Council on Cardiovascular Nursing; and the Interdisciplinary Council on Peripheral Vascular Disease. The American Academy of Neurology affirms the value of this statement as an educational tool for neurologists. Stroke 2009;40:2276-2293 